\title{
Mutations in the gene encoding filamin B disrupt vertebral segmentation, joint formation and skeletogenesis
}

\author{
Deborah Krakow ${ }^{1-3,21}$, Stephen P Robertson ${ }^{4,21}$, Lily M King ${ }^{2}$, Timothy Morgan ${ }^{4}$, Eiman T Sebald ${ }^{1}$, \\ Cristina Bertolotto ${ }^{2}$, Sebastian Wachsmann-Hogiu ${ }^{5}$, Dora Acuna ${ }^{2}$, Sandor S Shapiro ${ }^{6}$, Toshiro Takafuta ${ }^{7}$, \\ Salim Aftimos ${ }^{8}$, Chong Ae Kim ${ }^{9}$, Helen Firth ${ }^{10}$, Carlos E Steiner ${ }^{11}$, Valerie Cormier-Daire ${ }^{12}$, \\ Andrea Superti-Furga ${ }^{13}$, Luisa Bonafe ${ }^{13}$, John M Graham Jr ${ }^{2,14}$, Arthur Grix ${ }^{15}$, Carlos A Bacino ${ }^{16}$, \\ Judith Allanson $^{17}$, Martin G Bialer ${ }^{18}$, Ralph S Lachman ${ }^{2,19}$, David L Rimoin ${ }^{2,14,20}$ \& Daniel H Cohn ${ }^{2,14,20}$
}

\begin{abstract}
The filamins are cytoplasmic proteins that regulate the structure and activity of the cytoskeleton by cross-linking actin into three-dimensional networks, linking the cell membrane to the cytoskeleton and serving as scaffolds on which intracellular signaling and protein trafficking pathways are organized (reviewed in refs. 1,2). We identified mutations in the gene encoding filamin B in four human skeletal disorders. We found homozygosity or compound heterozygosity with respect to stop-codon mutations in autosomal recessive spondylocarpotarsal syndrome (SCT, OMIM 272460) and missense mutations in individuals with autosomal dominant Larsen syndrome (OMIM 150250) and the perinatal lethal atelosteogenesis I and III phenotypes (AOI, OMIM 108720; AOIII, OMIM 108721). We found that filamin B is expressed in human growth plate chondrocytes and in the developing vertebral bodies in the mouse. These data indicate an unexpected role in vertebral segmentation, joint formation and endochondral ossification for this ubiquitously expressed cytoskeletal protein.
\end{abstract}

Morphogenesis in vertebrate organisms requires the integration of extracellular signals with alterations in the cellular cytoskeleton. Filamins regulate the organization of cytoskeletal F-actin into either parallel bundles or orthogonal gel networks ${ }^{3}$ and also mediate interactions between subcortical actin networks and transmembrane receptors to modulate cell-cell, cell-matrix and intracytoplasmic signal transduction ${ }^{1,2,4}$. Mammals have three filamin genes, FLNA, FLNB and FLNC. FLNA and FLNB seem to be ubiquitously expressed ${ }^{5,6}$; FLNC is predominantly expressed in muscle. Human filamin genes are highly similar with conserved exon-intron structure, and there is $\sim 70 \%$ homology at the protein level ${ }^{2,7}$. The filamin monomer comprises an $\mathrm{N}$-terminal actin binding domain $(\mathrm{ABD})$ followed by a series of $24 \beta$-sheet repeats that collectively bind many cytoplasmic and transmembrane proteins ${ }^{1,2}$. Filamins exist in vivo as dimers. Dimerization, leading to homo- and possibly heterodimer formation, is mediated by interactions between C-terminal sequences ${ }^{5,8,9}$. Mutations in FLNA produce a spectrum of X-linked malformation and osteochondrodysplasia syndromes. FLNA loss-of-function mutations are usually embryonically lethal in males and underlie a neuronal migration disorder in females ${ }^{10}$. Mutations producing structural changes in the protein lead to numerous developmental anomalies in the brain, skeleton and viscera ${ }^{11}$.

Recently the gene associated with SCT, an autosomal recessive disorder characterized by short stature and vertebral, carpal and tarsal fusions $^{12,13}$, was localized on chromosome 3p14 (ref. 14). These studies and further recombination mapping (data not shown) identified a 4.7-cM candidate region, which included a 1.4-Mb region of homozygosity containing 14 genes. Mutations were not found in the candidate genes $W N T 5 A^{14}, A S B 14$ and IL17RD (also known as SEF) in affected individuals from the linked families. The gene FLNB localizes to this interval and, considering the role of FLNA in skeletogenesis ${ }^{11}$, we looked for mutations in FLNB in four unrelated families with SCT.

${ }^{1}$ Department of Obstetrics and Gynecology and ${ }^{2}$ Ahmanson Department of Pediatrics, Cedars-Sinai Research Institute, 8700 Beverly Blvd., Los Angeles, California 90048, USA. ${ }^{3}$ Department of Obstetrics and Gynecology, David Geffen School of Medicine at UCLA, Los Angeles, California, USA. ${ }^{4}$ Department of Paediatrics and Child Health, University of Otago, Dunedin, New Zealand. ${ }^{5}$ MIST Institute, Cedars-Sinai Research Institute, Los Angeles, California, USA. ${ }^{6}$ Department of Physiology, Jefferson Medical College, Philadelphia, Pennsylvania, USA. ${ }^{7}$ Department of Clinical and Laboratory Medicine, Yamanashi Medical University, Yamanashi, Japan. ${ }^{8}$ Northern Regional Genetics Service, Auckland, New Zealand. 9 Instituto da Criança, Faculdade de Medicina da Universade São Paulo, Brazil. ${ }^{0}$ Department of Medical Genetics, Addenbrookes Hospital, Cambridge, UK. ${ }^{11}$ Medical Genetics Department, Medical Sciences School, State University of Campinas, Campinas, São Paulo, Brazil. ${ }^{12}$ Département de Génétique Médicale, Hôpital Necker-Enfants Malades, Paris, France. ${ }^{13}$ Division of Molecular Paediatrics, Centre Hospitalier Universitaire Vaudois, Lausanne, Switzerland. ${ }^{14}$ Department of Pediatrics, David Geffen School of Medicine at UCLA, Los Angeles, California, USA. ${ }^{15}$ The Permanente Medical Group, Sacramento, California, USA. ${ }^{16}$ Department of Molecular and Human Genetics, Baylor College of Medicine, Houston, Texas, USA. ${ }^{17}$ Eastern Ontario Regional Genetics Program, Ottawa, Canada. ${ }^{18}$ Department of Pediatrics, Schneider Children's Hospital at North Shore/NYU Medical Center, Manhasset, New York, USA. ${ }^{19}$ Department of Radiology, David Geffen School of Medicine at UCLA, Los Angeles, California, USA. ${ }^{20}$ Department of Human Genetics, David Geffen School of Medicine at UCLA, Los Angeles, California, USA. ${ }^{21}$ These authors contributed equally to this work. Correspondence should be addressed to D.K. (deborah.krakow@cshs.org) or S.P. (stephen.robertson@stonebow.otago.ac.nz). 
a

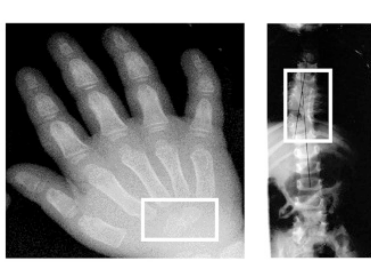

b

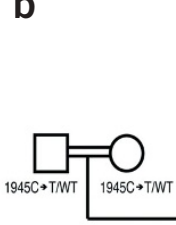

R99-438

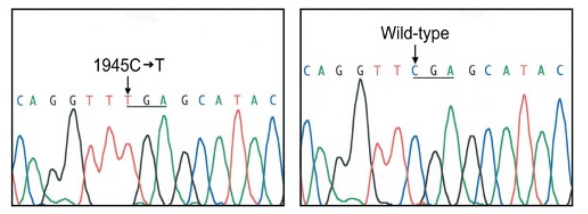

Individuals with SCT were either homozygous or compound heterozygous with respect to mutations in FLNB that resulted in stop codons (Fig. 1 and Table 1). Affected individuals in the consanguinous family R00-008 were homozygous with respect to the mutation 6408delC that predicts a translational frameshift and a stop codon four codons downstream. Affected individuals from two additional consanguinous families (R99-438 and R03-062) were homozygous with respect to mutations $(1945 \mathrm{C} \rightarrow \mathrm{T}$ and $7029 \mathrm{~T} \rightarrow \mathrm{G})$ predicted to create translational termination codons (R649X and Y2343X, respectively; Fig. 1). In one nonconsanguinous family (R00-084), the affected individual was compound heterozygous with respect to two mutations that predicted premature translation terminations at codons 818 and 1607. In all four families, the segregation of the mutations was compatible with the autosomal recessive inheritance pattern of SCT. All mutations found to underlie SCT predict premature stop codons within the repeat domain of filamin $\mathrm{B}$, and we therefore conclude that SCT results from absence or truncation of filamin B.

We next explored whether other skeletal dysplasias with vertebral fusions were associated with mutations in FLNB. Larsen syndrome is a genetically heterogeneous disorder characterized by multiple joint dislocations, craniofacial abnormalities and accessory carpal bones ${ }^{15-17}$ (Fig. 2). A gene associated with an autosomal dominant form of the disorder was localized to 3p21.1-14.1 (ref. 18). We studied four individuals with sporadically occurring Larsen syndrome and one family with a dominantly inherited form of the condition. We found heterozygosity with respect to missense mutations in FLNB that arose de novo in all five families (Fig. 2 and Table 1). Two mutations are predicted to lead to the amino acid substitutions $\mathrm{F} 161 \mathrm{C}$ and $\mathrm{E} 227 \mathrm{~K}$ in the $\mathrm{ABD}$ of filamin B. Three other mutations

predict a single amino acid deletion or substitution (1571delN, G1586R, G1691S) in repeats 14 and 15 of the protein.

AOI and AOIII are autosomal dominant lethal skeletal dysplasias with vertebral abnormalities, disharmonious skeletal maturation, poorly modeled long bones and joint dislocations ${ }^{19}$ (Fig. 3). The overlap of some of these clinical and radiographic features with Larsen syndrome $e^{20,21}$ prompted us to look for mutations in FLNB in individuals with these conditions. We identified mutations in FLNB in all three individuals with AOI and both individuals with AOIII that we studied (Fig. 3 and Table 1). All subjects were unrelated, and the phenotype arose sporadically in their families. The three individuals with AOI were heterozygous with respect to point mutations in FLNB that predicted single residue substitutions in the ABD. One individual with AOIII was heterozygous with respect to the same point mutation $(604 \mathrm{~A} \rightarrow \mathrm{G})$ identified in an individuals with AOI. Analysis of the available parental samples showed that one mutation associated with AOI and both mutations associated with AOIII occurred de novo. It has been suggested that Boomerang dysplasia is allelic with $\mathrm{AOI}^{22}$, but we found no mutations in FLNB in one individual with this diagnosis.

The diversity of skeletal abnormalities caused by mutations in FLNB indicate a central role for filamin B in skeletal morphogenesis. The presence of vertebral abnormalities (block fusions, vertebral hypoplasia, malsegmentation and clefting, posterior arch hypoplasia or aplasia) prompted us to examine expression of filamin $\mathrm{B}$ in mouse embryos by immunostaining. We observed intense, uniform filamin $B$ expression in condensing chondrocytes within vertebral bodies in sectioned embryos at embryonic day 14 (E14; Fig. 4). Reduced expression was evident in developing intervertebral discs. Additionally, there were a

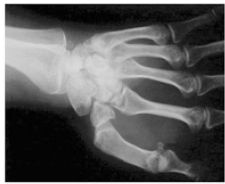

b

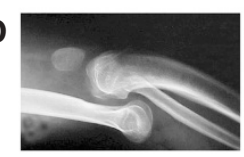

C

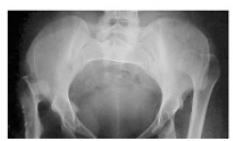

d
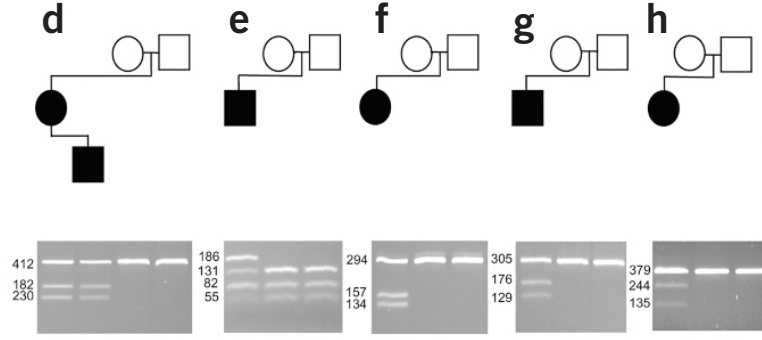

HpyCH4III

DNA

$482 T \rightarrow G$

Protein F161C

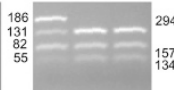

Bsp1286I

$679 G \rightarrow A$

E227K
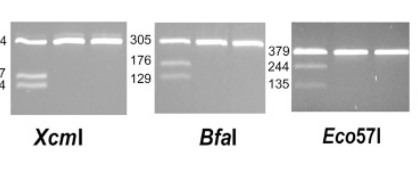

$(4756 \rightarrow A$

N1571del

G1586R

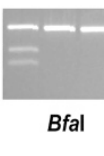

Figure 2 Mutations in FLNB are associated with Larsen syndrome. (a-c) Skeletal abnormalities in Larsen syndrome include supernumerary carpal bones and dislocations of the large joints, such as the knees and hips. (d-h) Pedigrees and restriction endonuclease digests show de novo mutations predicted to lead to amino acid substitutions in filamin B. The identities of the restriction endonucleases, the DNA mutation and the predicted protein sequence changes are shown below. Fragment sizes (in bp) are listed on the left. 
a

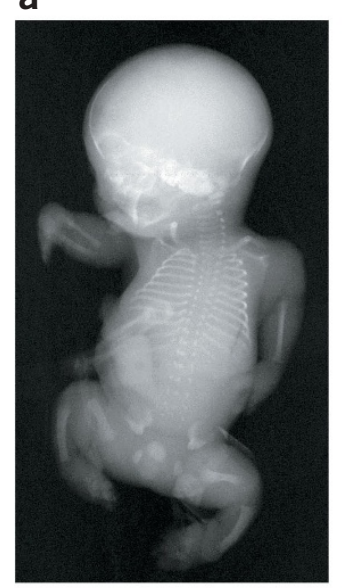

b
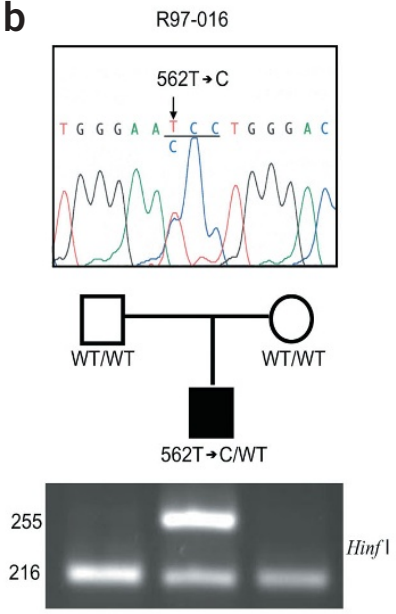

C
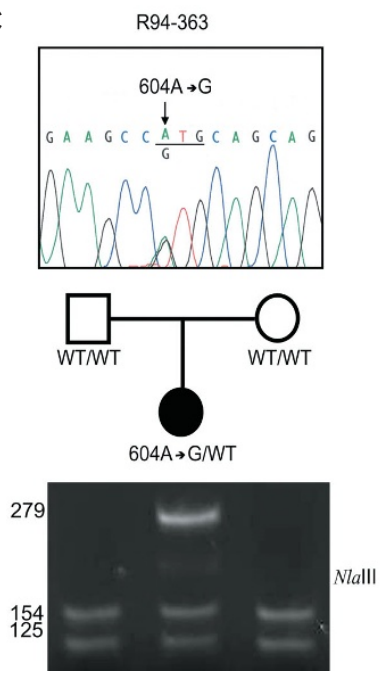

Figure 3 Mutations in FLNB in AOI and AOIII. (a) Anterior-posterior radiograph of an individual with AOI (from family R97-016) showing multiple skeletal abnormalities, including absent humeri, left radius, fibulae, hypoplastic femurs, absent thoracic vertebral bodies, a small thorax and lack of mineralization of the phalanges of the hands and feet. (b,c) Chromatographs show normal and mutated nucleotides in AOI (family R97-016) and AOIII (family R94-363), which are indicated by an arrow. The reading frame is underlined. The pedigrees and gel photographs of restriction endonuclease digestion products of PCR-amplified DNA from exon 3 show that mutations are de novo. Fragment sizes (in bp) are listed on the left and the restriction endonucleases used are indicated. WT, wild-type. detectable levels of filamin B in the kidney, lung, colon and cerebral periventricular region, as reported previously ${ }^{23}$.

The presence of short stature, epiphyseal delay and disharmonious bone mineralization in the group of disorders associated with mutations in FLNB suggest a role for filamin B within the epiphyseal growth plate. Some mutations in FLNA also affect stature and skeletal mineralization. We examined sections of normal fetal distal femur by immunofluorescence using antibodies directed against filamin A and filamin B. Filamin B was uniformly present throughout the growth plate, with expression detected in resting, proliferating, prehypertrophic and hypertrophic chondrocytes (Fig. 5a). In contrast, there was intense filamin A expression in chondrocytes in the hypertrophic zone, but diminished expression in resting and proliferating chondrocytes. Both filamins were localized to the cytoplasm, but in hypertrophic chondrocytes, filamin B was concentrated at the cell membrane whereas filamin A was distributed throughout the cytoplasm (Fig. 5a). In proliferating chondrocytes undergoing cell division, both filamins, but especially filamin B, were concentrated at the cleavage furrow between the dividing cells (Fig. 5b).

The demonstration that mutations in FLNB (Supplementary Fig. 1 online) are associated with a spectrum of autosomal dominant and recessive skeletal dysplasias indicates that Larsen syndrome, AOI and AOIII are genetically related conditions and adds SCT to the allelic series. Like mutations in FLNA ${ }^{11}$, mutations in FLNB produce a diversity of phenotypes, depending on the nature and location of the mutation. The premature termination mutations underlying SCT could lead to nonsense-mediated decay ${ }^{24}$ and absence of filamin B, but production of a stable truncated protein lacking a dimerization domain cannot be ruled out. Cell lines from the families with SCT were not available, and so these hypotheses could not be distinguished.

Dominant missense mutations in FLNB underlie Larsen syndrome, AOI and AOIII, disorders characterized by joint dislocation,

Table 1 Clinical findings and mutations in diseases associated with FLNB

\begin{tabular}{|c|c|c|c|c|c|c|c|c|c|c|c|c|}
\hline \multicolumn{9}{|c|}{ Family Phenotypic findings } & \multicolumn{4}{|c|}{ Mutation } \\
\hline $\begin{array}{l}\frac{n}{\omega 0} \\
\frac{0}{5} \\
\frac{\pi}{0} \\
\frac{\pi}{0}\end{array}$ & 亘 & 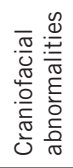 & 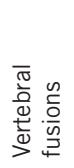 & 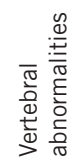 & 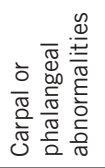 & 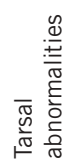 & 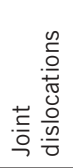 & 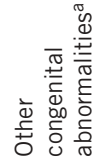 & ¿ & $\begin{array}{c}\text { 호 } \\
\text { జ }\end{array}$ & 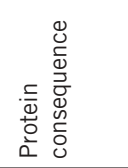 & 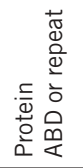 \\
\hline SCT & R99-438 & + & + & + & + & + & - & + & $1945 C \rightarrow T$ & 13 & R649X & 5 \\
\hline SCT & R03-062 & + & + & - & + & - & - & - & $7029 \mathrm{~T} \rightarrow \mathrm{G}$ & 43 & Y2343X & 22 \\
\hline \multirow[t]{2}{*}{ SCT } & R00-084 & - & - & - & + & - & - & - & $2452 C \rightarrow T$ & 16 & R818X & 6 \\
\hline & & & & & & & & & $4819 \mathrm{C} \rightarrow \mathrm{T}$ & 28 & $\mathrm{R} 1607 \mathrm{X}$ & 14 \\
\hline SCT & R00-008 & - & + & + & + & - & - & - & 6408delC & 39 & $\mathrm{~s} 2137 \mathrm{fs}$ & 20 \\
\hline Larsen & 319 & + & - & + & + & + & + & - & $482 \mathrm{~T} \rightarrow \mathrm{G}$ & 2 & F161C & $\mathrm{CHD} 2$ \\
\hline Larsen & 380 & + & - & - & + & + & + & - & $679 \mathrm{G} \rightarrow \mathrm{A}$ & 4 & E227K & $\mathrm{CHD} 2$ \\
\hline Larsen & 318 & + & - & + & + & + & + & - & 4711_4713delAAT & 28 & 1571delN & 14 \\
\hline Larsen & 334 & + & - & + & $U$ & $U$ & + & - & $4756 \mathrm{G} \rightarrow \mathrm{A}$ & 29 & G1586R & 14 \\
\hline Larsen & 225 & + & $U$ & $U$ & + & $U$ & + & - & $5071 \mathrm{G} \rightarrow \mathrm{A}$ & 31 & G1691S & 15 \\
\hline $\mathrm{AOI}$ & R95-326 & $U$ & - & + & + & $U$ & - & - & $518 \mathrm{C} \rightarrow \mathrm{T}$ & 2 & A173V & $\mathrm{CHD} 2$ \\
\hline $\mathrm{AOI}$ & R96-320 & + & + & + & $U$ & - & + & - & $604 \mathrm{~A} \rightarrow \mathrm{G}$ & 3 & M202V & $\mathrm{CHD} 2$ \\
\hline $\mathrm{AOI}$ & R97-016 & + & - & + & + & $U$ & + & - & $562 \mathrm{~T} \rightarrow \mathrm{C}$ & 3 & S188P & $\mathrm{CHD} 2$ \\
\hline AOIII & R83-120 & + & + & + & + & + & + & - & $2251 \mathrm{G} \rightarrow \mathrm{C}$ & 15 & G751R & 6 \\
\hline AOIII & R94-363 & + & - & + & + & + & + & - & $604 \mathrm{~A} \rightarrow \mathrm{G}$ & 3 & M202V & $\mathrm{CHD} 2$ \\
\hline
\end{tabular}

aOther congenital abnormalities include rarefication of the retinal vessels. U, unknown; CHD2, calponin homology domain 2; fs, frameshift. 


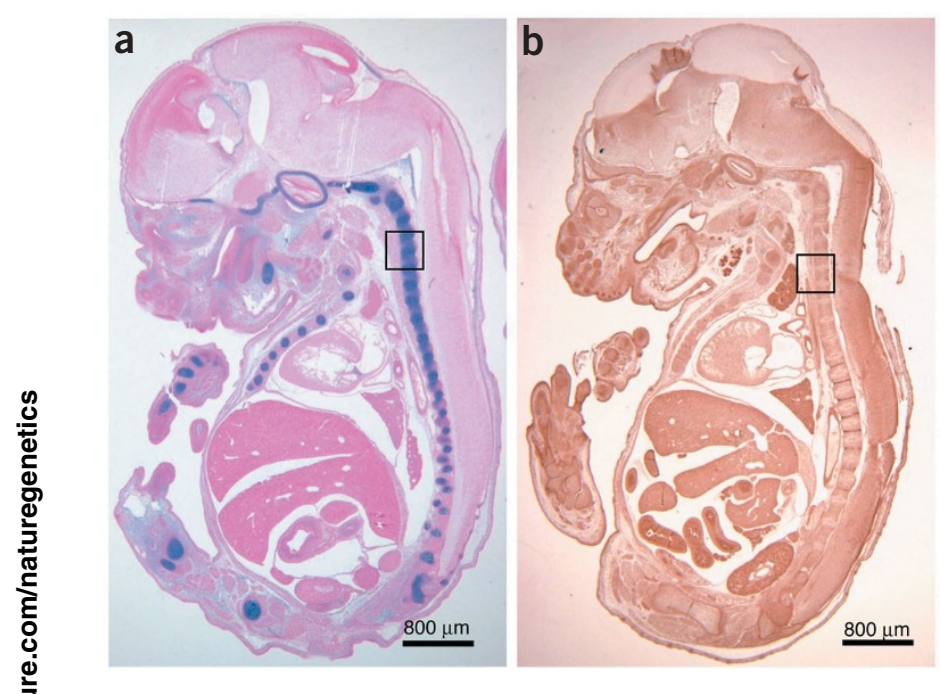

implicating filamin B in joint morphogenesis. Three of the four mutations associated with $\mathrm{AOI}$ or AOIII occurred in the $\mathrm{ABD}$, altering residues conserved among human paralogs, orthologous filamins from other species and other actin-binding proteins (Supplementary Fig. 2 online). The presence of the same mutation $(604 \mathrm{~A} \rightarrow \mathrm{G})$ in individuals with AOI and AOIII, conditions previously separated on grounds of radiology and chondro-osseus histology ${ }^{19}$, suggests that such distinctions do not reflect differences in their primary molecular pathogenesis.
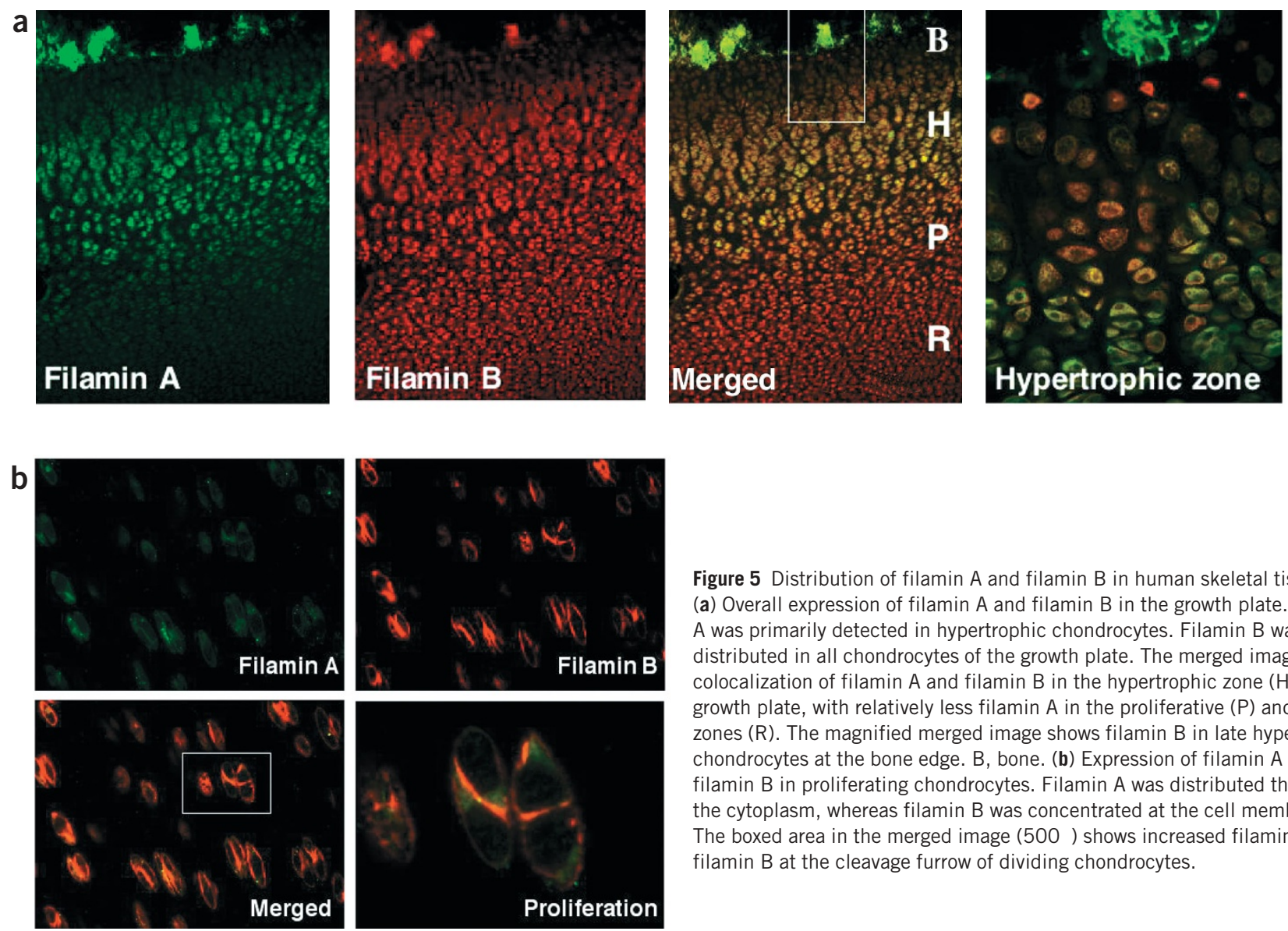
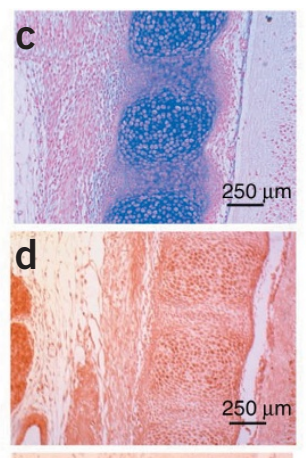

e

Three mutations producing Larsen syndrome ( 3 of 5 independent cases) altered conserved residues within repeats 14 and 15 . Whether these predicted substitutions disrupt specific binding interactions is unclear, as interacting partners for filamin B have not been identified for these repeat elements. As with disease phenotypes associated with mutations of the ABD of filamin $\mathrm{A}^{11}$, the severity of the disease associated with $A B D$ substitutions in the filamin $B$ is varied. Some well conserved residues corresponding to predicted helical components of the protein (A173V, M202V) produced lethal AOI or AOIII,
Figure 4 Distribution of filamin B in the E14 mouse embryo. (a) Sagittal section stained with alcian blue to detect chondrocytes. Nuclei section interrogated with antibody to filamin B and counterstained with hematoxylin. Brown regions correspond to filamin B staining. staining of chondrocytes in the developing vertebral bodies corresponding to the region with high levels of filamin B shown in $\mathbf{d}$.

(d) Boxed area from $\mathbf{b}$ showing high levels of filamin B in chondrocytes of the developing file embryo sagittal section. Area of the vertebral body corresponding to the boxed area from $\mathbf{b}$.

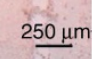

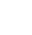


and substitution of a conserved residue distant from the major helices (F161C) produced Larsen syndrome. Whether a structural perturbation or alteration of an extrinsic interaction with filamin $B$ underlies these effects awaits clarification.

Female carriers of null mutations in FLNA manifest a neuronal migration disorder, periventricular nodular heterotopia ${ }^{10}$. Although both FLNA and FLNB are expressed in the periventricular region of the brain ${ }^{23}$, we are not aware of individuals with SCT who have seizures or other evidence for anomalous neuronal migration, indicating that presumptive loss-of-function mutations in FLNB do not have the same biological consequence as similar mutations in FLNA.

The demonstration of highly localized expression of filamin B in the cartilaginous condensations of developing vertebrae has not been previously appreciated and, together with the defects in vertebral development observed in individuals with mutations in FLNB, indicates a pivotal role for the protein in vertebral patterning and morphogenesis. In cartilage, distinct expression patterns for filamin A and filamin B indicate different roles for these proteins. Coexpression of the two proteins in hypertrophic chondrocytes suggests possible functional redundancy for the proteins in these cells, but there seems to be a distinct role for filamin B in the remainder of the growth plate. The histology of the growth plate in AOI and AOIII includes a diminished chondrocyte population and widespread acellular islands in the resting zone, sometimes containing cell remnants or multinucleated giant cells ${ }^{19}$. The observation of filamin B concentrated at the cleavage furrow of dividing chondrocytes may indicate that some mutations in FLNB confer a defect in cell division that leads to formation of multinucleated giant cells and possibly accelerated apoptosis, resulting in hypocellularity in the resting zone of the growth plate. Understanding the specific mechanisms by which FLNB mutations produce defects in skeletal morphogenesis and abnormalities in other organ systems will be facilitated by the findings described here. This will include the identification of specific filamin B binding partners and the pathways and downstream signal transduction targets that interact with this cytoskeletal protein during development.

\section{METHODS}

Linkage analysis. Under a protocol approved by the institutional review board, we obtained informed consent from all affected and unaffected individuals in the families studied. We collected blood and extracted DNA by standard protocols. Recombination mapping used the markers at the loci D3S2400, D3S3616, D3S1295, D3S1592, D3S2452, D3S1313 and D3S1234. Each 15- $\mu$ l PCR contained $25 \mathrm{ng}$ of genomic DNA, $200 \mu \mathrm{M}$ dNTPs, $0.33 \mu \mathrm{M}$ each primer, $0.5 \mathrm{U}$ of AmpliTaq Gold DNA polymerase (PE Biosystems) and $1.5 \mathrm{mM} \mathrm{MgCl}_{2}$. PCR was carried out using a Perkin Elmer 9700 GeneAmp Thermocycler as follows: 10 min at $95^{\circ} \mathrm{C} ; 35$ cycles of $94^{\circ} \mathrm{C}$ for $30 \mathrm{~s}, 55^{\circ} \mathrm{C}$ for $75 \mathrm{~s}$ and $72^{\circ} \mathrm{C}$ for $15 \mathrm{~s}$; and 10 min at $72^{\circ} \mathrm{C}$. We resolved PCR products by capillary electrophoresis using an ABI model 3100 automated DNA sequencer. We analyzed genotypes using the ABI Genotyper 2.5 software package. In each family, haplotypes were created by parsimony.

Mutation detection in FLNB. We amplified genomic DNA fragments by PCR using a Perkin Elmer 9700 GeneAmp Thermocycler. Primer sequences and conditions are available on request. We detected mutations using denaturing high performance liquid chromatography (Transgenomic) and subsequent automated sequence analysis or by direct sequence analysis alone. We subjected amplified fragments to sequence analysis using the ABI Prism Big Dye version 3 sequencing kit (Applied Biosystems) and separated the products by electrophoresis using an ABI model 377 automated DNA sequencer. We processed the sequence data using ABI software and analyzed it using Sequencher (Gene Codes). We confirmed mutations by bidirectional sequencing. After identifying a mutation in an affected individual, we carried out segregation analysis in families by sequence analysis, restriction endonuclease cleavage or single-strand conformation polymorphism analysis. We confirmed declared parental relationships by the segregation of four unlinked microsatellite markers (data not shown).

Antibody detection in mouse embryos. We removed the paraffin from sections of mouse embryos (Novagen) and hydrated them through a series of xylene and alcohol washes. Antigen retrieval was carried out by standard microwave citrate methods and then quenched in $0.3 \% \mathrm{H}_{2} \mathrm{O}_{2}$ for $30 \mathrm{~min}$. We blocked slides in $10 \%$ goat serum, $1 \%$ bovine serum albumin, $0.5 \%$ Triton in phosphate-buffered saline (PBS) for $30 \mathrm{~min}$ at room temperature and then incubated them with a 1:200 dilution of antibody to filamin B directed against the $\mathrm{H} 1$ epitope ${ }^{6}$ in $10 \%$ goat serum, $1 \%$ bovine serum albumin, $0.5 \%$ Triton in PBS overnight at $4{ }^{\circ} \mathrm{C}$. We stained slides with immunoperoxidase using the Vectastain ABC Elite Kit (Vector Laboratories) and mounted them in Permount (Fisher Scientific). For alcian blue staining, we stained the mouse E14 section with 1\% Alcian blue (Sigma) in 3\% acetic acid for $45 \mathrm{~min}$ and counterstained it for $5 \mathrm{~min}$ with $0.1 \%$ nuclear fast red (Biomedical Specialties) in $5 \%$ aluminum sulfate. We dehydrated the slides with alcohol and xylene washes and then mounted them in Permount. We analyzed the results with an Olympus Fluoview confocal microscope (Scientific Instruments Company). We captured images with an Apogee KX-2E digital camera (Apogee Instruments) and processed them with an Image-Pro, Focus Control computer program for a PC computer (Scientific Instruments Company).

Growth plate immunofluorescence. We fixed the distal fetal femur from a normal 20-week-old fetus in formalin and then decalcified it in 3\% EDTA diluted in $0.1 \mathrm{M}$ PBS. We cut $70-\mu \mathrm{m}$ sections using a vibratome, blocked sections for $1 \mathrm{~h}$ in $5 \%$ donkey or goat serum in $0.1 \%$ Triton in PBS and then incubated them with a primary monoclonal antibody to filamin A (Neomarkers) and/or a polyclonal antibody to filamin B, each at a 1:200 dilution in 5\% serum in PBS, at $4{ }^{\circ} \mathrm{C}$ for $3 \mathrm{~d}$. We washed sections, treated them with either Alexa Fluor 488-conjugated donkey antibody to mouse IgG or Alexa Fluor 568-conjugated goat antibody to rabbit IgG (Molecular Probes) at a 1:200 dilution in 5\% normal serum in PBS at room temperature for $1 \mathrm{~h}$. We washed the sections and mounted them on slides using VECTASHIELD (Vector Laboratories) with DAPI. Duplicates of each section were incubated with PBS instead of the primary antibody as a negative control. We analyzed sections using a fluorescence microscope as described above and with a Leica TCS SP confocal microscope (Leica Microsystems).

Note: Supplementary information is available on the Nature Genetics website.

\section{ACKNOWLEDGMENTS}

We thank all the families who participated in this research; M. Priore, G. Rose and F. Field of the International Skeletal Dysplasia Registry for their assistance in collecting the families; N. Ehtesham for her assistance with preparing the figures; and $\mathrm{K}$. Lyons for discussions regarding the expression of filamin B during mouse development. This work was supported in part by grants from the US National Institute of Health (to D.H.C., D.K. and D.L.R.), the Cedars-Sinai General Clinical Research Center, the Drown Foundation (to D.K.) and the National Organization for Rare Disorders (to J.M.G.). D.H.C. is the recipient of a Winnick Family Foundation Clinical Scholars award. S.P.R. and T.M. are supported by the Child Health Research Foundation of New Zealand.

\section{COMPETING INTERESTS STATEMENT}

The authors declare that they have no competing financial interests.

Received 6 November 2003; accepted 29 January 2004

Published online at http://www.nature.com/naturegenetics/

1. Stossel, T.P. et al. Filamins as integrators of cell mechanics and signaling. Mol. Cell Biol. 2, 138-145 (2001)

2. Van der Flier, A. \& Sonnenberg, A. Structural and functional aspects of filamins. Biochim. Biophys. Acta 1538, 99-117 (2001).

3. Niederman, R., Amrein, P.C. \& Hartwig, J. Three-dimensional structure of actin filaments and of an actin gel made with actin-binding protein. J. Cell Biol. 96, 1400-1413 (1983).

4. Loy, C.J., Sim, K.S. \& Yong, E.L. Filamin-A fragment localizes to the nucleus to regulate androgen receptor and coactivator functions. Proc. Natl. Acad. Sci. USA 100, 4562-4567 (2003).

5. Gorlin, J.B. et al. Human endothelial actin-binding protein (ABP-280, nonmuscle filamin): a molecular leaf spring. J. Cell Biol. 111, 1089-1105 (1990). 
6. Takafuta, T., Wu, G., Murphy, G.F. \& Shapiro, S.S. Human $\beta$-filamin is a new protein that interacts with the cytoplasmic tail of glycoprotein Ibo. J. Biol. Chem. 273, 17531-17538 (1998).

7. Chakarova, C. et al. Genomic structure and fine mapping of the two human filamin gene paralogues FLNB and FLNC and comparative analysis of the filamin family. Hum. Genet. 107, 597-611 (2000).

8. Sheen, V.L. et al. Filamin A and filamin B are co-expressed within neurons during periods of neuronal migration and can physically interact. Hum. Mol. Genet. 11 2845-2854 (2002).

9. Himmel, M., Van Der Ven, P.F., Stocklein, W. \& Furst, D.O. The limits of promiscuity: isoform-specific dimerization of filamins. Biochemistry 42, 430-439 (2003).

10. Fox, J.W. et al. Mutations in filamin 1 prevent migration of cerebral cortical neurons in human periventricular heterotopia. Neuron 21, 1315-1325 (1998).

11. Robertson, S.P. et al. Localized mutations in the gene encoding the cytoskeletal protein filamin A cause diverse malformations in humans. Nat. Genet. 33, 487-491 (2003).

12. Honeywell, C., Langer, L. \& Allanson, J. Spondylocarpotarsal synostosis with epiphyseal dysplasia. Am. J. Med. Genet. 109, 318-322 (2002).

13. Steiner, C.E., Torriani, M., Norato, D.Y. \& Marques-de-Faria, A.P. Spondylocarpotarsal synostosis with ocular findings. Am. J. Med. Genet. 91, 131-134 (2000).

14. Steiner, C.E. et al. A locus for spondylocarpotarsal synostosis syndrome at chromosome 3p14. J. Med. Genet. (in the press).

15. Larsen, L.J., Schottdstaedt, E.R. \& Bost, F.C. Multiple congenital dislocations associated with characteristic facial abnormality. J. Pediatr. 37, 574-584 (1950)

16. Latta, R.J., Graham, C.B., Aase, J., Scham, S.M. \& Smith, D.W. Larsen's syndrome: a skeletal dysplasia with multiple joint dislocations and unusual facies. J. Pediatr. 78,
291-298 (1971).

17. Marques, M.J. Larsen's syndrome: clinical and genetic aspects. J. Genet. Hum. 28 , 83-88 (1980).

18. Vujic, M. et al. Localization of a gene for autosomal dominant Larsen syndrome to chromosome region 3p21.1-14.1 in the proximity of, but distinct from, the COL7A1 locus. Am. J. Hum. Genet. 57, 1104-1113 (1995).

19. Sillence, D., Worthington, S., Dixon, J., Osborn, R. \& Kozlowski, K. Atelosteogenesis syndromes: a review, with comments on their pathogenesis. Pediatr. Radiol. 27, 388-396 (1997).

20. Nishimura, G., Horiuchi, T., Kim, O.H. \& Sasamoto, Y. Atypical skeletal changes in otopalatodigital syndrome type II: phenotypic overlap among otopalatodigital syndrome type II, boomerang dysplasia, atelosteogenesis type I and type III, and lethal male phenotype of Melnick-Needles syndrome. Am. J. Med. Genet. 73, 132-138 (1997).

21. Schultz, C., Langer, L.O., Laxova, R. \& Pauli, R.M. Atelosteogenesis type III: Iong term survival, prenatal diagnosis, and evidence for dominant transmission. Am. J. Med. Genet. 83, 28-42 (1999).

22. Wessels, M.W. et al. Prenatal diagnosis of boomerang dysplasia. Am. J. Med. Genet. 122, 148-154 (2003)

23. Sheen, V.L. et al. Mutations in the X-linked filamin 1 gene cause periventricular nodular heterotopia in males as well as in females. Hum. Mol. Genet. 10, 1775-1783 (2001).

24. Maquat, L.E. Nonsense-mediated RNA decay in mammalian cells: A splicing-dependent means to down-regulate the levels of mRNAs that prematurely terminate translation. in Translational Control of Gene Expression (Sonenberg, N., Hershey, J.W.B. \& Mathews, M.B. eds.) 849-868 (Cold Spring Harbor Press, Cold Spring Harbor, New York, 2000). 